\title{
Inflammatory Pain Study in Animal Models
}

Created by: Yean Chun Loh ${ }^{1}$, Yean Chun Loh ${ }^{2}$, Mun Fei Yam ${ }^{3}$, Chuan Wei Oo

4, Rusliza Basir 5

1, School of Chemical Sciences, Universiti Sains Malaysia.; nicklesloh@hotmail.com

2, School of Chemical Sciences, Universiti Sains Malaysia.; lohyeanchun@usm.my

3, School of Pharmaceutical Sciences, Universiti Sains Malaysia; yammunfei@usm.my

4, School of Chemical Sciences, Universiti Sains Malaysia; oocw@usm.my

5, Faculty of Medicine and Health Sciences, Universiti Putra Malaysia; rusliza@upm.edu.my

Version received: 22 June 2020

check for

updates

Pain is an easily recognized sensation that is experienced by humans and animals alike. However, the process behind the production of the pain experience is a complex pathway that requires parallel integration of both the emotional and sensory experiences together with noxious perceptual information registered by multiple layers of our brain structure with the purpose of defending our body from harm's way. Here, the complete protocol that is being adapted for inflammatory pain study in animals induced by different phlogogenic agents and different assessment methods were elaborated along with the underlying mechanism of actions. This provides a concise idea and improves our scientists' understanding of inflammatory pain management in future research.

\section{Basic Mechanisms of Pain}

Generally, there are three main events will occur in the pain mechanism due to noxious stimulation which includes transduction, transmission and modulation of the signals. These signals will be conducted in two ways, where the upward carrying sensory information from the body to the brain via the spinal cord is known as ascending, and the signals sent from the brain to the reflex organs through the spinal cord is defined as descending pathway. Primarily, both the peripheral nervous system (PNS) and central nervous system (CNS) is involved in all types of pain perception. PNS composed of ganglia and nerves that located outside the brain and spinal cord, playing vital roles in connecting the CNS to our limbs and organs. Whereas the CNS that comprises of the spinal cord and brain is mainly functioning in integrating and interpreting the signals sent from the PNS, then immediately coordinating all the activities in our body [1]. Here, the analgesics that refer to agents that are used to relieve pain will act through the CNS or PNS mechanism pathway without significantly affecting consciousness. Analgesics can be narcotic or non-narcotic. Narcotic means that the analgesics that act through CNS but do not produce an anti-inflammatory response, such as tramadol and morphine, whereas non-narcotic will act peripherally whilst producing an anti-inflammatory effect such as non-steroidal anti-inflammatory agents (NSAIDs).

\section{Inflammatory Pain Study in Animal Models}

Inflammatory pain in animal models has been widely used and accepted as the study model to understand the mechanisms of tissue injury-induced persistent pain. The animal models of tissue injury and inflammatory hyperalgesia can be provoked by various inflammatory agents. Amongst them, the CFA and $\lambda$ carrageenan are the most frequently used for the purpose of animal inflammatory pain studies. However, there are still no existing models that could potentially simulate all the symptoms of inflammatory pain. 


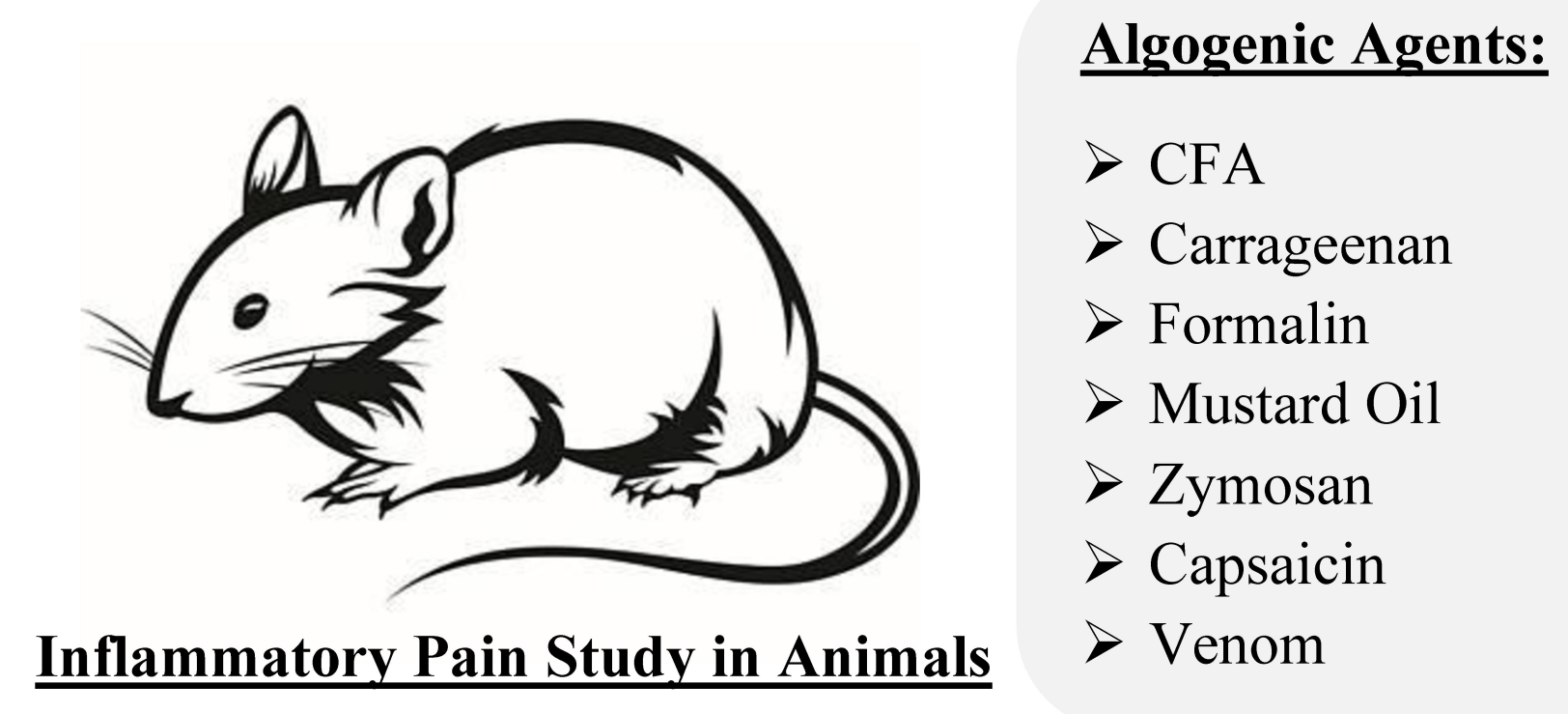

\section{Assessment Methods:}

$\checkmark$ Weight Bearing (CatWalk)

$\checkmark$ Gait \& Posture Analysis

$\checkmark$ Spontaneous Mobility

$\checkmark$ Thermal and Mechanical Sensitivity of Paws

$\checkmark$ Struggle Threshold Angle

$\checkmark$ Vocalization Threshold

$\checkmark$ Plethysmometer \& Micrometer Measurements

$\checkmark$ Grimace Scale Test

\section{Complete Freund's Adjuvant}

Previously, the polyarthritic and persistent pain studies in animals were developed by inoculating the rodent's tail in the Mycobacterium butyricum oil suspension. However, this model is not frequently being used due to the concomitant illness that typically develops in rodents following the polyarthritic induction test. Generally, CFA is a well-known, dosedependent inflammatory agent that is normally injected into the footpad of animals to provoke a local inflammation response and a persistent pain in the subjects within minutes to hours post-injection, with the peak effect observed at about 6 to $8 \mathrm{~h}[2][3]$. In rats, approximately $30-200 \mu \mathrm{g}$ of CFA is injected in the hind paw of the animals and it can result in extreme edema within $24 \mathrm{~h}$ and both the peaks of hyperalgesia and allodynia can be observed within $5 \mathrm{~h}$ postinjection lasting for a minimum of 2 weeks 4$]$. These phenomena mimic rheumatoid arthritis occurring in humans ${ }^{[5]}$. From the previous studies executed, Fischer 344 (FIS) rats demonstrated a higher thermal hyperalgesia sensation in comparison to the Lewis (LEW) and Sprague Dawley (SD) subtype of rats based on the computational scoring differences by subtracting the paw withdrawal latency (PWL) of the contralateral paw from the CFA-injected paw. The major disadvantage of this model study is that the rats will experience minimal weight reduction with normal grooming behavior [2]. 


\section{Carrageenan Model}

Generally, there are three main types of carrageenan available such as the iota, kappa and lambda. The lambda form is commonly used to induce acute inflammatory responses in animal models due to its gel state in room temperature. Typically, the intraplantar injection of 100 or $25 \mu \mathrm{L}$ of $1 \%(\mathrm{w} / \mathrm{v}$, suspension in saline) $\lambda$ carrageenan to the rat or mouse paw, respectively, to cause a local inflammatory effect with two phases (biphasic) of paw edema development observed. The first phase of paw edema will occurs in the rat within the first 30 min post-injection due to the release of pro-inflammatory factors including serotonin, bradykinin, histamine and prostaglandins, whereas the second phase will begin at the end of the first hour post-injection and lasts until the third hour, with its peak being observed within 3-5h of carrageenan injection due to the attribution of neutrophil infiltration, nitric oxide and continuing prostaglandin

generation [6][7]. However, the early phase of paw edema happening in the mouse will last for $6 \mathrm{~h}$ and followed by the second phase response that peaks at about $72 \mathrm{~h}$. On the other hand, the injection of carrageenan will induce the formation of thermal hyperalgesia which peaks on the third day and 4th-hour post-injection, respectively. Thermal hyperalgesia typically will last for almost $96 \mathrm{~h}$ once it is induced. The cardinal signs will be resolved completely within 2-3 days which is apparently a much shorter duration as compared to the CFA-induced inflammatory model. According to previous studies, the FIS rats demonstrated the highest significance in terms of thermal hyperalgesia compared to LEW and SD rats upon $3.5 \mathrm{mg}$ of carrageenan administration. The proposed mechanisms involved in carrageenan-induced thermal hyperalgesia and mechanical allodynia is mediated via norepinephrine and serotonergic pathways to exhibit analgesic response, whereas the anti-inflammatory action of analgesic drugs on the carrageenaninduced edema is mediated via the down-regulation of both the substance $P$ and prostaglandin $E_{2}$.

\section{Formalin Model}

This test is predominantly used to study acute and long-lasting pain induced in the paw of rats or mice and it is considered as more satisfactory model of clinical pain to screen analgesic drugs $[8]$. Formalin, which is $37 \%$ formaldehyde, will be used as the pain-inductor. There are studies that utilized chemicals like ethylene diamine tetraacetic acid (EDTA), complete Freund's adjuvant (CFA), capsaicin, hypertonic saline, or bee venom; however, they are less likely used as the algogens for pain study. The site of injection and the dosage of pain-inducing agent to be administered are strictly dependent on the purpose of the study. Typically, a small volume of 5\% (v/v) formalin will be injected into the soft cutaneous tissue which is usually the dorsal surface of the plantar surface of the hind paw rather than the forelimb [9]. The reason behind the choice of the hind paw as the location for injecting the pain-inducing agent is to avoid the walking patterns of the animal to be affected by the presence of the fluid in the injection site.

Formalin-provoked pain can induce three main behavioral responses-phasic flexion, tonic flexion, and licking of the injected limb. In the intraplantar injections of the formalin in the paw of mice or rats, there will be a biphasic nocifensive behavior; the first phase of nocifensive response will occur within 5 min post-injection of formalin lasting up to $10 \mathrm{~min}$. There is a quiescent phase (sensitization) following the first phase of nocifensive reaction where the subject shows relatively lesser pain responses for a period of $10 \mathrm{~min}$. Then, the second phase of responses will start on the $15^{\text {th }}$ minute and lasts for a rough estimation of 40 to $60 \mathrm{~min}$. Fundamentally, the first phase of response is related to the direct stimulation of nociceptors such as C-fibre and low-threshold mechanoreceptors including the up-regulation of substance $P$, while the second phase is involving central sensitization of the rodents due to the inflammatory phenomena within the dorsal horn neurons including the up-regulation of serotonin, histamine, prostaglandin and bradykinin. The formalin test is commonly tested using the weight-score method of behavioral rating, which can be assessed on four scales based on the posture of the rodents (0: normal posture, 1: injected paw stays on the ground but not supporting the body, 2: withdrawal of injected paw, and 3: licking, shaking, or nibbling of injected paw). The response demonstrated by the subjects will be marked down continuously per unit of time or at regular time intervals. Once the test is completed, the animal should be administered with an analgesic drug leading to the gradual diminishing of the pain-evoked response for the next $2 \mathrm{~h}$ and the gradual recovery of the inflammation site of the injected area within 7 to 10 days. The formalin test is sensitive to centrally acting analgesic agents. 


\section{Zymosan and Mustard Oil Models}

Both zymosan and mustard oil can induce inflammation with hyperalgesia response via the activation of TRPV channels, which will cause excitatory effects on the primary afferent nociceptors ${ }^{110]}$. However, the effects of both these inflammatory agents are relatively short and only can last for up to $20 \mathrm{~min}$. The topical application of mustard oil to the lateral surface of the rat's hind paw can induce slight edema and plasma extravasation, whilst frequent biting and vocalizations can be observed from the tested subject. This phenomenon can last up to 7 min post-application. The application of mustard oil can facilitate the tail-flick test by enhancing the rate of reflex within 5 min, reaching its peak at approximately $20 \mathrm{~min}$ of post-application [11]. Application of zymosan intraplantarly can result in a time-dependent and persistent thermal and mechanical hyperalgesia response. Typically, the mechanical hyperalgesia will appear upon the application of more than $1.25 \mathrm{mg}$ of zymosan, peaking on the 4th hour in rats. Thermal hyperalgesia will occur in two phases: the early and late phase. The early phase peaks at $30 \mathrm{~min}$ post-application if $\geq 2.5 \mathrm{mg}$ of zymosan is applied, whereas the late phase peaks at the $4^{\text {th }}$ hour upon application of $\geq 0.0625 \mathrm{mg}$ of $z y m o s a n$. Furthermore, edemas will appear if $\geq 2.5 \mathrm{mg}$ of zymosan is applied, and will reach its peak at 30 minutes postadministration. The duration of responses is highly dependent on the dose of zymosan applied. Additionally, the higher dosage such as $\geq 5 \mathrm{mg}$ of zymosan will cause a spontaneous pain in the rodents.

\section{Capsaicin Model}

The application of the capsaicin will stimulate nociceptors to cause a neurogenic inflammation via activation of the TRPV1 channels. Typically, hyperalgesia, allodynia, and flare reactions will potentially appear upon the injection of capsaicin intradermally in rats $(10 \mu \mathrm{g} / 10 \mu \mathrm{L}$ in $10 \%$ ethanol and 2-hydroxypropyl BETA cyclodextrin). For instance, the primary hyperalgesia induced by capsaicin towards a punctuate stimuli covers a larger area than those reacting to a stroking stimuli, followed by the flare response will be observed and extended to secondary hyperalgesia ${ }^{[12]}$. The visual flare will occur within seconds of capsaicin post-injection whereas the hyperalgesia occurs immediately upon injection, reaches its peak within 15 to $30 \mathrm{~min}$ and lasts for $21 \mathrm{~h}$ in the case of rats. The same goes for the hyperalgesia reaction towards stroking stimuli, the peak will appear at about 15 min but only last for a maximum of 6 $\mathrm{h}$. The inflammatory effects induced by capsaicin are dose-dependent and may vary according to the area of the subject being injected. Additionally, the intraplantar injection of capsaicin in the rat's paw can provoke neurogenic inflammation that persists for $4 \mathrm{~h}$ and thermal hyperalgesia that persists for 45 min maximum ${ }^{[13]}$.

\section{Bee Venom Model}

This test was first developed by ${ }^{[14]}$ to study the nociceptive effects in rats. In this test, the bee venom will be injected subcutaneously into the hind paw of the rats. Subsequently, the nocifensive behaviors such as lifting, flinching, or licking of the injected paw will be observed with it usually persisting up to $2 \mathrm{~h}$, followed by thermal hyperalgesia, mechanical allodynia and edema development within 72-96 $\mathrm{h}$ post-injection. In addition to that, the thermal hyperalgesia could appear in the contralateral hind paw as well. The duration of spontaneous pain-related behavior responses is dose-dependent in both time course and response intensity. This test was claimed to be sensitive to pharmacological intervention by non-steroidal anti-inflammatory drugs and morphine in demonstrating the analgesic effects. The onset and duration of action of different inflammation-inducing agents were summarized in Table 1 below.

Table 1. The onset and duration of action elicited by different inflammatory agents and its sensitization caused. 


\begin{tabular}{|c|c|c|c|c|c|}
\hline $\begin{array}{l}\text { Inflammatory } \\
\text { Agents }\end{array}$ & $\begin{array}{l}\text { Quantity } \\
\text { Applied }\end{array}$ & Hyperalgesia & Allodynia & $\begin{array}{l}\text { Onset } \\
\text { of } \\
\text { Action } \\
\text { (within) }\end{array}$ & $\begin{array}{l}\text { Duration } \\
\text { of } \\
\text { Action } \\
\text { (s) }\end{array}$ \\
\hline $\begin{array}{l}\lambda \\
\text { Carrageenan }\end{array}$ & $\begin{array}{l}100 \mu \mathrm{L} \text { of } 1 \% \\
(w / v)\end{array}$ & $+(\mathrm{t})$ & $+(\mathrm{m})$ & $30 \mathrm{~min}$ & 3 days \\
\hline Formalin & $\begin{array}{l}50 \mu \mathrm{L} \text { of } 5 \% \\
(v / v)\end{array}$ & + & + & $5 \mathrm{~min}$ & $60 \mathrm{~min}$ \\
\hline $\begin{array}{l}\text { Complete } \\
\text { Freund's } \\
\text { Adjuvant }\end{array}$ & $\begin{array}{l}1: 1 \text { dilution in } \\
\text { phosphate } \\
\text { buffered } \\
\text { saline }\end{array}$ & + & + & $5 \mathrm{~h}$ & 2 weeks \\
\hline Mustard Oil & $\begin{array}{l}0.0625-\geq 5 \\
\mathrm{mg}\end{array}$ & + & + & $5 \min$ & $60 \min$ \\
\hline Zymosan & D & $+(\mathrm{t} / \mathrm{m})$ & + & $30 \mathrm{~min}$ & $24 \mathrm{~h}$ \\
\hline Capsaicin & $\begin{array}{l}10 \mu \mathrm{g} / 10 \mu \mathrm{L} \text { in } \\
10 \% \text { Etol and } \\
2- \\
\text { hydroxypropyl } \\
\text { BETA } \\
\text { cyclodextrin }\end{array}$ & $+(\mathrm{t} / \mathrm{m})$ & + & $1 \mathrm{~min}$ & $21 \mathrm{~h}$ \\
\hline Venom & D & $+(\mathrm{t})$ & $+(\mathrm{m})$ & $1 \mathrm{~min}$ & $96 \mathrm{~h}$ \\
\hline
\end{tabular}

Notes: -: to; +: Presence; D: Depends on the types of agents used; Etol: ethanol; m: mechanical; t: thermal.

\section{Inflammatory Pain Assessment Methods in Animal Models}

The measurements of the inflammatory pain in animal models are strictly dependent on the behavioral changes on the animals, including the foot posture and gait analysis, dynamic and static weight bearing, thermal and mechanical sensitivity of the paw, and the spontaneous mobility [15].

\section{Weight Bearing}

The weight bearing test is commonly used as the main assessment on the measurement of inflammatory pain in arthritic models. Initially, the restrained animal will be placed in an angled Plexiglas chamber and the force exerted by each hind limb will be detected and measured (in grams) by the incapacitance tester over an average time of 5 s. Besides that, the stepping force of each limb can also be measured by using the force sensor plates while the animal is allowed to walk through an enclosed walkway. The walking pattern of the animal across the walkway is recorded by 
a camera, which is fixed at the bottom of the transparent glass floor. The digitized output and the simultaneously videotaped images will be synchronized manually in order to obtain the peak vertical weight bearing by each limb. Eventually, the force exerted by each limb will be expressed as the percentage of either their body weight or the sum of the force exerted by both hind paws. The stepping force difference between each hind limb will further be calculated as a ratio for clearer comparison [16]. Furthermore, the stepping force exerted by each limb across the glass floor can also be evaluated by the gait analysis system, also known as "CatWalk". A white fluorescent tube will be used as the indicator for the point of contact by the paw of the animals while the animals walk through the corridor, and the intensity of the resulting illumination will be measured. At the same time, a wide-angle CCD camera is positioned under the corridor to record the walking patterns. The major concern of this test is that the voluntary (motivation) of the animals to walk across the walkway. If the subjects refuse to walk across the path, there will be no results generated.

\section{Gait and Posture Analysis}

Fundamentally, the combination of gait and posture analysis is frequently used for pain-related functional impairment studies [17]. Before the experiment starts, an electrode is attached to the plantar surface of each hind paw. After that, the animal is placed on $30 \mathrm{~cm}$ diameter stainless steel cylinder which will be rotating at 4 rpm in order to force the animal to walk. The circuit is terminated once the electrode is in contact with the cylindrical floor, whilst the time of the circuit remained closed is recorded. The behavioral signs that could be included during this test are foot aversion, non/partial weight bearing, toes curling, prevention of contact with the limb. Eventually, the ratio of the contact time of the control foot to the affected foot is calculated as well as the paw elevation time.

\section{Spontaneous Mobility}

This test is commonly used for knee joint arthritis pain models by studying the locomotor activity using activity boxes or the biotelemetry system. The biotelemetry system is frequently used to assess the spontaneous mobility and the temperature of the body in the animals. Initially, the transmitter of the biotelemetry system is implanted in the peritoneal cavity of the animal, and a receiver is placed under the cage. The locomotor activity of the animal will cause the transmitter to release a signal which is relayed by the consolidation of matrix into the peripheral processor which is connected to a computer. Subsequently, radio waves and the activity are received as counts by the receiver [18]. Another method has been used for spontaneous mobility assessment in animals; that is, activity boxes which are made of photobeam and phototransistors. The phototransistors will be placed on the wall opposite to the photobeam, which will be activated once the animal's movements have interrupted the beam. The photobeam patterns and the frequency emitted through the movement of the animal will be subsequently recorded as an activity score.

\section{Thermal and Mechanical Sensitivity of Paws}

Both Randall-Selitto and Von Frey filaments tests are used to evaluate the mechanical sensitivity of the animal's paw with knee joint arthritis. Apart from this, both the paw withdrawal and hot plate test are also used for assessing the thermal sensitivity of arthritis rodent's paw. Refer to Randall-Selitto, Von Frey filaments, paw withdrawal, and hot-plate test mentioned above. The significant reduction of both the withdrawal latency and withdrawal threshold of the affected limb will be observed in this test $[19]$.

\section{Struggle Threshold Angle}

The knee extension angle can be used to evaluate the mechanical sensitivity of arthritic knee in the study subjects. Initially, the animal is gently held upward by using one hand, and the tibia of the animal is extended to a point where the subject shows signs of struggling. The distance of the heel of the foot during the extension is measured, and the angle of the extension is calculated. Typically, the struggle threshold angle of the arthritis knee will be significantly 
reduced compared to the control [20].

\section{Vocalization Threshold}

In this test, the vocalization threshold is measured by compressing the arthritic knee of the restrained animals using a pair of calibrated forceps. Both the audible and ultrasonic vocalizations (approximately $2 \mathrm{~min}$ ) are detected and measured simultaneously upon pre- and post-stimulation by using a recording chamber integrated with a computerized analysis system. The ultrasonic vocalization of the rodents represents its emotional response, whereas the audible vocalization is a representation of its nocifensive response. Typically, the frequency used for the detection of the audible range is $20-16 \mathrm{kHz}$, whereas the ultrasonic range is $29-21 \mathrm{kHz}$. The duration and rate of vocalization will be increased in animals suffering from knee joint arthritis. Eventually, the vocalization during stimulation (VDS) and vocalization after discharge (VAD) will be interpreted individually.

\section{Plethysmometer and Micrometer Measurements}

Both of these devices are used to assess the edema as a surrogate of pain induced by the algogens in the paw of the rodents (rats or mice) as well as to evaluate the effectiveness of anti-edematous agents [21]. The measurement principle applied in Plethysmometer is based on Archimedes Law by measuring the fluid's displacement upon the immersion of the rat's paw in the measuring vessel, the displacement of the fluid is reflected to the exact volume of rat's paw swelling. Typically, the fluid (mercury or water) displacement will range from 0 to $0.5 \mathrm{~mL}$ for mice and 0 to 7 $\mathrm{mL}$ for rats, whereas the micrometer is a simple device that used directly to measure the thickness of the rat's paw edema. There are different types of enhanced Plethysmometer being used by scientists to evaluate the rat's paw edema; however, the same principle is applied.

\section{Grimace Scale Test}

The grimace scale is a standardized behavioral coding system specially designed for animal pain studies that involve the noxious stimuli of moderate duration (within $10 \mathrm{~min}$ to $4 \mathrm{~h}$ ) whilst accompanied with facial expressions caused by pain. The animals will be placed in Plexiglas chamber with a digital video camera (preferred high resolution) positioned at either end of the chamber. Then, the video camera will be set to record $30 \mathrm{~min}$ before and after the noxious stimulation applied, typically zymosan or CFA, to capture the facial expressions of the animals for grimace scoring purpose. Manually, the experimenter will screen, randomly scramble and score the face images of the animals before being told that which group of the animals has been treated. This manual image selection method was replaced by the automated frame capture using the Rodent Face Finder. After selection, each image will be given a score of 0 (not present), 1 (moderately visible) or 2 (severe) based on the facial features described in [22], then the mean grimace scores will be calculated as average score across the action units [23]. Generally, the grimace scale will quantify the facial changes of the animals (rats or mice) in a number of "action units", and this number is directly proportional to the stimulus intensity. There are five facial features (action units) can be observed in both mice and rats including the orbital tightening, nose bulge, cheek bulge, whisker change and ear position change as described by [22]. However, there is a major exception in the cheek or nose because the bulging occurs naturally in rats, but not in mice [24]. The noxious stimuli that are applied superficially will yield a lower score compared to deep tissues such as viscera. The advantages of this test are that no preliminary animal training or special equipment is required and the measurements of the behavioral response from the animals are spontaneously emitted by themselves. In addition, the major drawbacks of this test are the labor-intensive nature of the frame grabbing in the case of manual operation and the limited

duration of the pain expressions from the animals. This test is frequently used for morphine-like analgesics studies.

\section{References}

1. Mun Fei Yam; Yean Chun Loh; Chu Shan Tan; Siti Khadijah Adam; Nizar Abdul Manan; Rusliza Basir; General Pathways of Pain 
Sensation and the Major Neurotransmitters Involved in Pain Regulation. International Journal of Molecular Sciences 2018, 19, 2164, 10.3390/ijms19082164.

2. M J ladarola; L S Brady; G Draisci; R Dubner; Enhancement of dynorphin gene expression in spinal cord following experimental inflammation: stimulus specificity, behavioral parameters and opioid receptor binding.. Pain 1988, 35, 313-326.

3. M. J. Millan; A. Czlonkowski; B. Morris; Christoph Stein; R. Arendt; A. Huber; V. Höllt; A. Herz; Inflammation of the hind limb as a model of unilateral, localized pain: influence on multiple opioid systems in the spinal cord of the rat. Pain 1988, 35, 299-312, 10.1016/0304-3959(88)90140-6.

4. Li-Xing Lao; Rui-Xin Zhang; Grant Zhang; Xiaoya Wang; Brian M. Berman; Ke Ren; A parametric study of electroacupuncture on persistent hyperalgesia and Fos protein expression in rats. Brain Research 2004, 1020, 18-29, 10.1016/j.brainres.2004.01.092.

5. M E Rosenthale; A comparative study of the Lewis and Sprague Dawley rat in adjuvant arthritis.. Archives internationales de pharmacodynamie et de therapie 1970, 188, 14-22.

6. R Vinegar; W Schreiber; R Hugo; Biphasic development of carrageenin edema in rats.. Journal of Pharmacology and Experimental Therapeutics 1969, 166, 96-103.

7. Charles A. Winter; Edwin A. Risley; George W. Nuss; Carrageenin-Induced Edema in Hind Paw of the Rat as an Assay for Antiinflammatory Drugs. Experimental Biology and Medicine 1962, 111, 544-547, 10.3181/00379727-111-27849.

8. J.P. Dzoyem; Lyndy J McGaw; V. Kuete; Udo Bakowsky; Anti-inflammatory and Anti-nociceptive Activities of African Medicinal Spices and Vegetables. Medicinal Spices and Vegetables from Africa 2017, 2017, 239-270, 10.1016/b978-0-12-809286-6.00009-1.

9. Janel M. Boyce-Rustay; Prisca Honore; Michael F. Jarvis; Animal Models of Acute and Chronic Inflammatory and Nociceptive Pain. Advanced Structural Safety Studies 2010, 617, 41-55, 10.1007/978-1-60327-323-7_4.

10. Diana M. Bautista; Sven-Eric Jordt; Tetsuro Nikai; Pamela R. Tsuruda; Andrew J. Read; Jeannie Poblete; Ebenezer N. Yamoah; Allan I. Basbaum; David Julius; TRPA1 Mediates the Inflammatory Actions of Environmental Irritants and Proalgesic Agents. Cell 2006, 124, 1269-1282, 10.1016/j.cell.2006.02.023.

11. M.O. Urban; M.C. Jiang; G.F. Gebhart; Participation of central descending nociceptive facilitatory systems in secondary hyperalgesia produced by mustard oil. Brain Research 1996, 737, 83-91, 10.1016/0006-8993(96)00631-2.

12. R H LaMotte; C N Shain; D A Simone; E F Tsai; Neurogenic hyperalgesia: psychophysical studies of underlying mechanisms.. Journal of Neurophysiology 1991, 66, 190-211.

13. Hart D. Gilchrist; Brandon L. Allard; Donald A. Simone; Enhanced withdrawal responses to heat and mechanical stimuli following intraplantar injection of capsaicin in rats. Pain 1996, 67, 179-188, 10.1016/0304-3959(96)03104-1.

14. William R. Lariviere; Ronald Melzack; The bee venom test: a new tonic-pain test. Pain 1996, 66, 271-277, 10.1016/03043959(96)03075-8.

15. Rachel Combe; Steve Bramwell; Mark J. Field; The monosodium iodoacetate model of osteoarthritis: a model of chronic nociceptive pain in rats?. Neuroscience Letters 2004, 370, 236-240, 10.1016/j.neulet.2004.08.023.

16. Mun Fei Yam; Lip Yee Por; Kok Khiang Peh; Mariam Ahmad; Mohd Zaini Asmawi; Lee Fung Ang; Delina Beh Mei Yin; Sim Ying Ong; Muthanna Fawzy Abdulkarim; Ghassan Zuhair Abdullah; et al.Ibrahim Muhammad SalmanOmar Ziad AmeerElsnoussi Ali Hussin MohamedMohd Akmal HashimElham FarsiSook Yee Hor Development of a Stepping Force Analgesic Meter for a Rat Arthritic Model. Sensors 2011, 11, 5058-5070, 10.3390/s110505058.

17. Carlos Rogério Tonussi; Sergio H. Ferreira; Rat knee-joint carrageenin incapacitation test: an objective screen for central and peripheral analgesics. Pain 1992, 48, 421-427, 10.1016/0304-3959(92)90095-s.

18. P. Gegout-Pottie; L. Philippe; M.-A. Simonin; C. Guingamp; P. Gillet; Patrick Netter; B. Terlain; Biotelemetry: an original approach to experimental models of inflammation. Inflammation Research 1999, 48, 417-424, 10.1007/s000110050481.

19. Kathleen A. Sluka; K. N. Westlund; Behavioral and immunohistochemical changes in an experimental arthritis model in rats. Pain 1993, 55, 367-377, 10.1016/0304-3959(93)90013-f.

20. Yun Cho Yu; Sung-Tae Koo; Chang Hoon Kim; Yeoungsu Lyu; James J Grady; Jin Mo Chung; Two variables that can be used as pain indices in experimental animal models of arthritis. Journal of Neuroscience Methods 2002, 115, 107-113, 10.1016/s01650270(02)00011-0.

21. D Green; J Haines; A rat paw plethysmometer for single-handed operation.. Medical and biological engineering 1966, 4, $285-287$.

22. Dale J Langford; Andrea L Bailey; Mona Lisa Chanda; Sarah E Clarke; Tanya E Drummond; Stephanie Echols; Sarah Glick; Joelle Ingrao; Tammy Klassen-Ross; Michael L LaCroix-Fralish; et al.Lynn MatsumiyaRobert E SorgeSusana G SotocinalJohn M TabakaDavid WongArn M. J. M. Van Den MaagdenbergMichel D FerrariKenneth D. CraigJeffrey S. Mogil Coding of facial expressions of pain in the laboratory mouse. Nature Methods 2010, 7, 447-449, 10.1038/nmeth.1455.

23. Titilola Akintola; Charles Raver; Paige Studlack; Olivia Uddin; Radi Masri; Asaf Keller; The grimace scale reliably assesses chronic pain in a rodent model of trigeminal neuropathic pain. Neurobiology of Pain 2017, 2, 13-17, 10.1016/j.ynpai.2017.10.001.

24. Susana G Sotocinal; Robert E. Sorge; Austin Zaloum; Alexander H Tuttle; Loren J Martin; Jeffrey S. Wieskopf; Josiane Mapplebeck; Peng Wei; Shu Zhan; Shuren Zhang; et al.Jason J McDougallOliver D. KingJeffrey S. Mogil The Rat Grimace Scale: A partially automated method for quantifying pain in the laboratory rat via facial expressions. Molecular Pain 2011, 7, 55-55, 10.1186/17448069-7-55. 


\section{Keywords}

Inflammation; Animal pain; Neurological; CatWalk; Grimace Scale; Phlogogenic agent

(c) (i) (C) 2020 by the author(s). Distribute under a Creative Commans CC BY license 